Horizons philosophiques

\title{
Considérations sur l'interprétation chez Vladimir Jankélévitch et Igor Stravinsky
}

\section{Emmanuelle Corbel}

Volume 16, numéro 1, automne 2005

Raisonner la musique

URI : https://id.erudit.org/iderudit/801306ar

DOI : https://doi.org/10.7202/801306ar

Aller au sommaire du numéro

Éditeur(s)

Collège Édouard-Montpetit

ISSN

1181-9227 (imprimé)

1920-2954 (numérique)

Découvrir la revue

Citer cet article

Corbel, E. (2005). Considérations sur l'interprétation chez Vladimir Jankélévitch et Igor Stravinsky. Horizons philosophiques, 16(1), 74-85.

https://doi.org/10.7202/801306ar d'utilisation que vous pouvez consulter en ligne.

https://apropos.erudit.org/fr/usagers/politique-dutilisation/ 


\section{Considérations sur l'interprétation chez Vladimir Jankélévitch et Igor Stravinsky}

La fréquentation régulière et constante des concerts classiques, afin de découvrir ou redécouvrir sans cesse du répertoire et surtout de nouveaux interprètes, fait apparaître de nombreuses questions. Comment rendre compte d'une interprétation où nous avons été émerveillés, touchés, émus ou tout au contraire, où nous sommes restés de marbre, insatisfaits devant une perte de repères dans la reconnaissance de l'œuvre, une écriture devenue incompréhensible. Comment faire partager et formuler de la façon la plus explicite ce que nous pouvons ressentir à la lecture d'une œuvre musicale aussi bien en tant qu'auditeur qu'en tant que praticien? Parler de la musique ou sur la musique semble ne pas aller de soi. Convertir en phrases justes l'œuvre entendue, révélée, intimement liée à la temporalité et surtout, à l'irréversibilité de l'instant, semble bien délicat tant elle est indéniablement rattachée à l'interprétation de la partition. Quel discours et quel langage existent-t-ils pour parler de ce qui reste lié à l'exécution? II semble alors légitime de rapprocher les propos de Vladimir Jankélévitch et d'Igor Stravinsky aux points de vue presque opposés en apparence, puisqu'ils se sont intéressés aux problèmes de langage et d'interprétation dans la musique en prenant des chemins différents. Néanmoins, ces deux auteurs apportent des éclairages non négligeables dans la compréhension des méandres et des subtilités de l'interprétation. Ces deux personnalités ont eu des parcours professionnels très différents : le premier, Vladimir Jankélévitch ${ }^{1}$, philosophe-pianiste a voué sa vie à alterner au sein de ses ouvrages philosophiques des réflexions esthétiques et musicologiques provenant du déchiffrage d'un nombre considérable de partitions à son piano ; le deuxième, Igor Stravinsky² est à la fois compositeur et pianiste-interprète de ses propres œuvres. II a par ailleurs publié des ouvrages qui serviront de référence à cette analyse: Chroniques de ma vie, Poétique musicale et les entretiens accordés à Robert Craft ${ }^{3}$. Bien que ces écrits aient donné lieu à des travaux mettant en cause leur authenticité dans la rédaction, nous renvoyons le lecteur concernant ce point à l'article de Valérie Dufour 4 et nous nous référerons aux éditions citées ci-dessus ainsi qu'à la volonté réelle de ce compositeur de réfléchir sur ces différents problèmes musicaux ${ }^{5}$. 
En confrontant les réflexions d'un philosophe-musicien et celles d'un compositeur, il semblait risqué, voire même incompatible, d'établir une synthèse à la fois enrichissante pour la musique et pour l'esthétique. Pourtant en parcourant les écrits de Vladimir Jankélévitch et les ouvrages d'Igor Stravinsky, on remarque des préoccupations communes concernant la musique, le message qu'elle contient - si elle en contient un - et la réception de celui-ci par les auditeurs, le public. Un même souci constant sur le problème de l'interprétation jalonne consciemment ou inconsciemment les lignes écrites par ces deux auteurs aux références, aux goûts musicaux et aux horizons professionnels éloignés. II est donc nécessaire de faire le point, d'apporter un regard nouveau sur ce que l'on appelle interprétation par le biais tout d'abord des écrits sur la musique de Vladimir Jankélévitch puis, par les réflexions rassemblées par Igor Stravinsky et provenant de ses expériences de compositeur et d'interprète pianiste. Enfin, il s'agit de s'attarder sur l'utilité qu'il peut y avoir d'émettre une interprétation et sur celle "d'émettre un discours interprétatif sur les interprétations entendues».

Prenant conscience qu'à l'issue d'un concert ou après la lecture d'une œuvre, une vive curiosité intellectuelle mêlée d'étonnement s'impose, Vladimir Jankélévitch s'est mis en quête d'expliciter ce qu'il a pu ressentir, constater et entendre. Pour cela, sa démarche et ses recherches tentent de mieux faire comprendre ses instants passés avec la musique et d'en rendre compte 6 . Ce qui frappe en premier lieu, dans les écrits de Vladimir Jankélévitch, c'est la comparaison constante entre langage musical et langage verbal. Pour cela, il emploie une terminologie, un vocabulaire abondant toujours affiné, toujours repris sous sa plume, afin de reconsidérer le mystère de la musique se manifestant dans l'exécution musicale. Ce philosophe-musicien est à la recherche d'un absolu qui apparaît et disparaît, qui est un problème limite, un «je-ne-sais-quoi et un presque-rien". C'est autour de ce thème central qu'il élabore une réflexion profonde sur laquelle il revient sans cesse. De cette expérience musicale vécue, vivante, unique et irréversible, l'esthétique de Vladimir Jankélévitch est une recherche du «je-nesais-quoi', de l'infini, de l'inaccessible, de l'absolu, tentant de relater et de formuler le mystère contenu au sein de la musique où la connaissance se fait difficilement accessible 7 . II tente donc de mieux comprendre le rapport que la musique entretient avec le langage 
verbal et le langage qui la constitue, et plus précisément avec l'ineffable. Aussi, dans la pensée de Vladimir Jankélévitch, la musique française plus que les autres, constituerait-elle pour ce philosophe, l'accès à quelque chose de caché voire d'inexprimable ${ }^{8}$. En opérant comme cela, il s'est forgé un style bien à lui et inimité jusqu'à ce jour. Le vocabulaire employé comprenant "l'ineffable», "l'indicible», "l'inexprimable", "l'équivoque", "l'inachevé», n'est pas choisi au hasard, mais demeure au premier abord pour le lecteur, flou et intrigant?.

Finalement, Vladimir Jankélévitch ne tente peut-être pas d'expliquer la musique mais élabore plutôt un discours sur elle, dans le but de renvoyer à son expression même. II tente de comprendre, de rendre compte du pouvoir d'évocation et de suggestion de la musique par une terminologie au service du message qu'il voulait laisser. Son discours vise, d'une part, à persuader ses lecteurs de l'utilité, de la richesse et de l'apport de la musique à l'homme et, d'autre part, à rendre compte de l'épaisseur du mystère contenu dans son expression sachant qu'elle peut être rejouée et "s'interpréter" à l'infini ${ }^{10}$ »... Par cette méthode, par ce discours, quel accent veut-il mettre, faire comprendre et apporter à son lecteur? Son discours relativement technique a néanmoins ses limites, devient lui-même un "organe obstacle" parce qu'il reste quelque chose d'enfoui au sein même de la musique, quelque chose de réfractaire à toute formulation provenant de la musique elle-même rattachée au temps, à la temporalité et à l'instant. La musique, domaine réfractaire au discours, à la conceptualisation, peut alors mener à des discours loin de la réalité musicale; cela explique en partie l'abondance des exemples musicaux parcourant l'ensemble de l'œuvre de ce philosophe. Toutes ces références sont à la fois le témoignage d'une immense culture musicale, d'une connaissance approfondie et indéniable de cette discipline, et prouvent également qu'au delà de tout discours, la musique reste plus importante que tout. La musique elle-même limite alors tout discours. L'œurre a et garde sans aucun doute son sens en elle même. Le discours s'impose après le jeu ou l'écoute d'une œuvre, mais toute analyse selon lui, n'est qu'un passage, une étape avant de retourner à l'œuvre elle-même. C'est pourquoi, comme il l'explique lui-même lors d'une interview, il s'exprime plus sur la musique qu'il ne l'explique ${ }^{11}$. N'oublions pas, cependant que d'un point de vue strict, la musique garde une part mystérieuse parce qu'elle ne peut être signifiée. En effet, il n'existe pas de parallélisme 
strict, de traduction littérale entre le son et le sens. La phrase musicale est et sera toujours différente de la phrase grammaticale. La signification reste ainsi cachée en musique, ce qui constitue toute sa singularité et tout son mystère. Tout reste à dire, comme le souligne Vladimir Jankélévitch ${ }^{12}$, allant jusqu'à comparer la musique à une figure de style : la litote. La musique en fait, dit peu pour en révéler beaucoup. Elle n'a rien à prouver, à démontrer et ne s'exprime que par des symboles et des analogies, donnant finalement moins qu'elle ne promet. Elle suggère surtout, se revêt de charme : elle signifie sans désigner, sans expliquer ${ }^{13}$. Elle promet quelque chose qui ne se clarifie pas car elle est naturellement équivoque. Vladimir Jankélévitch n'explicite pas littéralement, mais formule seulement un de ces sens possibles. II s'interroge, dégage des éléments de réponses, essaie de comprendre pour mieux justifier son retour à la musique. C'est ainsi qu'il renvoie à l'écoute et à la pratique de la musique. Il est impossible de mentionner ici tous les thèmes se rapprochant du langage de la musique et de l'ensemble de ses réflexions sur le mystère, sur l'absolu de cette discipline artistique. Sa pensée, riche et toute en nuances, accorde une part importante au temps et à la temporalité. II nous laisse de magnifiques pages sur l'émotion, part subjective de ce moment unique, où l'instant devenu révélation devient un irremplaçable souvenir teinté par la suite de nostalgie, qu'il ne se prive pas de mentionner à diverses reprises dans ses travaux ${ }^{14}$. Dans la problématique qu'il s'agit de poursuivre dans cette étude, il est donc essentiel de retenir pour Vladimir Jankélévitch que la musique contient un mystère - précisément au sein de la création, au sein du langage musical, au sein de l'exécution - justifiant l'interprétation et le retour continuel et incessant à de nouvelles approches des grandes œuvres. Afin de mieux comprendre ce mystère et pour saisir le message que la musique nous livre dans l'éclair de l'instant, il effectue un important travail à partir d'un vocabulaire très riche pour relater son pouvoir de suggestion et d'évocation, ceci afin de nous permettre d'affiner les impressions qu'elle nous laisse.

Igor Stravinsky, à la différence de l'auteur sur lequel nous venons de tenter d'effectuer une synthèse, adopte au premier abord, un point de vue beaucoup plus strict. En effet, pour lui la musique n'exprime rien en soi. Elle est, selon lui, impuissante à exprimer quoi que ce soit 15 . Ce sont les mélomanes qui attendent beaucoup de la musique. Le plaisir et les émotions sont en fait extérieurs à l'essence de la musique, ineffable en elle-même. Si la musique n'exprime rien d'autre 
que les indications de la partition, c'est pour mieux donner l'impression, que seules les directives du compositeur sont importantes : il l'a lui-même prouvé en ayant recours à l'enregistrement ${ }^{16}$. Ainsi, laisse-til d'indélébiles informations pour exécuter l'ensemble de ses œuvres de façon propre tout en respectant sa volonté. Cette démarche lui permet de laisser un héritage précieux aux futures générations et lui assure, pour ceux qui auront écouté ses enregistrements, le bon déroulement dans le déchiffrage et la lecture de ses œuvres. La musique n'est pas, selon lui, autre chose que ce qu'elle est, et spéculer sur elle est une démarche vaine. À la suite de cette remarque, on comprend mieux pourquoi il a attaché autant d'importance à vouloir définir, distinguer le rôle d'un exécutant et le rôle d'un interprète.

En effet, Igor Stravinsky attend avant tout d'un musicien d'être un bon exécutant. Là encore si l'on devait lui demander de donner son avis sur l'importance entre le compositeur et l'exécutant, il est certain que le premier l'emporte sur le second, mais il ne peut nier l'importance de l'exécutant pour donner vie aux œuvres des compositeurs ${ }^{17}$. Là, intervient la notion d'interprétation dérangeante, selon lui, parce que cette notion sous-entend des limites imposées à l'exécutant ou qu'il s'impose pour transmettre la musique à l'auditeur 18 . Tandis que la notion d'exécution implique, comprend dans sa réalisation la stricte volonté explicite d'épuiser dans son application ce qu'elle ordonne ${ }^{19}$. II nous précise cependant, qu'il reste dans un texte, même bien écrit, des éléments secrets qui ne s'expliquent pas, difficilement analysables et définissables. Sur ce point, il rejoint ce que Vladimir Jankélévitch a tenté de chercher, de formuler, dans un «je-ne-sais-quoi» et un «presque-rien». Là, commence l'importance du rôle de l'interprète usant de son intuition et de son talent; ainsi, semble-t-il, se séparent l'exécutant et l'interprète par une différence de niveau. Selon lgor Stravinsky, l'exécutant se soumet au texte tandis que l'on attend de l'interprète, qu'il fasse une traduction du texte, qu'il fasse de «la perfection de cette traduction matérielle, une complaisance amoureuse ${ }^{20}$ ". Cependant, il émet quelques réserves et indique les attentes et les «limites" d'un bon interprète. Celui-ci doit avant tout être un infaillible exécutant : «Le secret de la perfection réside avant tout dans la conscience de la loi que lui impose l'œuvre qu'il exécute 21 ». Un interprète se doit de faire preuve de soumission, de souplesse, de maîtrise technique, de 
culture et de sens de la tradition. II ajoute et commente que les vrais interprètes ont une expression brillante de virtuosité, une soumission et une modestie du geste ainsi qu'une sobriété de l'expression qui fait d'eux des artistes de race. Mais attention, Igor Stravinsky n'hésite pas à faire part de la perversion et des pièges cachés derrière ce rôle enviable en apparence. L'interprète ne doit pas trop mettre sa personnalité et sa vanité en avant. Certains selon lui, cherchent ou recherchent un succès immédiat, la commodité d'une fructueuse carrière... tandis que les " bons " interprètes sont ceux qui servent la musique avec loyauté22. Un bon interprète se doit, selon lui, de ne pas rendre l'interprétation fantaisiste, c'est-à-dire qu'elle ne doit pas être sous l'emprise de considérations extra-musicales fausses et ce personnage ne doit pas trop s'inspirer d'éléments extrinsèques à la musique qui déformeraient l'interprétation du texte. Si bien qu'avec ces conseils, il constitue les limites et les qualités d'un bon interprète. Sa position assez radicale et presque contradictoire vis à vis du texte musical dont il prétend qu'il ne veut rien exprimer, s'adoucit alors avec cette analyse et ces précisions ${ }^{23}$. Finalement, ces personnages exécutants, interprètes... ont un rôle essentiel, représentent les valeurs profondes de la musique, ont une considérable éducation de l'oreille et de l'esprit24, savent respecter leurs limites et doivent tenir compte du fait que la communication de l'œuvre passe par l'oreille mais aussi par la vue, par le fait de suivre le bon geste 25 ... II est temps maintenant de rassembler ces considérations et d'examiner les points communs de ces deux auteurs et l'apport de leurs réflexions, de leurs discours dans le domaine de l'interprétation.

Ces deux avis, aussi divergents soient-ils, se rejoignent au sujet de l'interprétation, de sa difficulté et par là même, du rôle essentiel de son existence, par celui qui lui donne vie : l'exécutant, l'interprète. Toutefois, il est nécessaire de rappeler que Vladimir Jankélévitch a tenu à insister sur l'équivoque du texte musical. Aussi l'interprétation nous place-t-elle devant une recherche de sens, de clarification, d'univocité. En tentant de rendre compte, d'apporter quelques solutions à la singularité du phénomène musical, il rend légitime le bien fondé de toutes les interprétations. II renvoie la musique à la question du pluralisme où une même cause peut produire une infinité d'effets... Finalement, il interroge, dégage des éléments de réponses, essaie de comprendre pour mieux justifier son retour à la musique, pour nous permettre de constater qu'il n'y a pas un rapport réglé entre la signification et le symbole, entre une idée et une métaphore. Cela 
justifie l'emploi d'un vocabulaire abondant utilisé par Vladimir Jankélévitch et accentue son rapport avec la musique, de préférence plus poétique que scientifique.

Ces deux auteurs mettent l'accent sur l'équivoque du texte musical26. Mais assigner un sens ou du sens, c'est reconnaître qu'un texte possède une part d'obscurité ou d'ambiguitté propre au domaine de l'interprétation. II existe donc indéniablement un phénomène d'interprétation dès l'instant où l'on décrypte un ensemble de signes ne possédant pas par lui-même un caractère d'évidence suffisant. La question qui s'en suit, est de savoir ce que l'on entend et ce que l'on attend d'une interprétation de qualité. Igor Stravinsky établit un rapport entre interprétation et lecture... Pour lui, la musique n'exprime rien, il veut laisser à celle-ci tout son art, sa légitimité et préfère éviter toutes les dérives du mot interprétation. Selon lui, une interprétation suppose avant tout une bonne lecture, une sorte de traduction. Cependant, en constatant que le sens des choses ne va pas de soi, il faut donc une transposition d'un langage dans un autre. En conséquence, une interprétation comprend une part d'élucidation motivée, justifiée, comprise par l'interprète et l'auditeur. Elle apporte une part supplémentaire, oriente et enrichit le sens propre. L'interprète intervient sur un texte, produit par un autre sujet (le compositeur) qui n'est pas là pour en rendre compte. En ce sens, l'interprétation vise à donner de la rationalité, à apporter un raisonnement cohérent, à combler un déficit de sens. Interpréter, ce n'est pas seulement expliquer mais cela conduit à l'essence de la partition par la vision d'un être singulier.

Interpréter constitue pour celui qui joue ce rôle tout à la fois d'élucider, de mettre à découvert l'implicite dans le texte musical, mais aussi d'exécuter, de rendre effectif, de donner à entendre en optimisant la pure lisibilité de cette œuvre et surtout de transmettre, d'entrer en communication par l'intermédiaire de cette pièce avec un public, des auditeurs... Ce concept d'interprétation pose donc problème parce qu'il sous entend que l'interprète va porter l'obscur du texte musical à la transparence, à une plus grande proximité. L'interprète tente de toucher du doigt, de s'approcher le plus possible du mystère caché de la partition. II tente de donner à entendre, d'optimiser et de révéler la part obscure contenue dans le texte musical pour la mener vers la lumière. C'est pourquoi lgor Stravinsky insiste sur le fait que l'exécution doit être la plus propre possible. Or, souvent l'interprète pense trop à l'interprétation, entretient par là sa réputation, sa marque personnelle avec un certain égocentrisme ${ }^{27}$. 
Finalement le but d'interpréter est-il de fixer le sens, de l'arrêter? II s'agit plus exactement de révéler quelque chose de profondément humain, essentiel et tellement proche de la réalité humaine ${ }^{28}$.

Ainsi, le langage musical amène-t-il à émettre une interprétation qui est redoublée en quelque sorte par toute tentative de discours ou de conceptualisation. Aussi, afin d'éviter de s'égarer dans une interprétation arbitraire de son texte musical, Igor Stravinsky établit-il une conservation de ses intentions et directives par le biais d'enregistrements effectués par la maison Pleyel29. En dehors de cette solution, à quel moment peut-on émettre un discours sur la musique? Trop tôt, il est ou se résume à une recherche d'éléments biographiques, se noie dans des détails de sources et d'événements plus ou moins importants ou en rapport avec la musique concernée ${ }^{30}$. Si le discours arrive trop tard, il transforme, décompose la musique en procédés techniques, ce qui a été création pure, miraculeuse et gratuite ou du moins est-ce ainsi qu'elle apparaît ou qu'elle est perçue et ressentie, sortie des contraintes socio-historiques pesant sur les compositeurs, et ce, à toutes les époques. Mais, comme le rappelle Vladimir Jankélévitch, un discours ne peut remplacer la sensation de celui qui la fait vivre sous ses doigts, ne peut remplacer le désir de l'interprète de jouer, de créer ou de recréer devant nous une œuvre ${ }^{31}$. Le danger est de formuler soit un discours complémentaire à la musique elle-même et dans ce cas trop éloigné d'elle (de sa réalité), soit un discours avec explications de ses moindres détails menant à un irrationalisme. Mais, au delà des réflexions que l'on peut apporter du point de vue historique et sociologique, c'est tout le problème de l'éducation des individus à la musique abordé ici en arrière plan par Igor Stravinsky. II souligne combien la musique et cela, quelles que soient les spéculations que l'on puisse faire sur elle, demande un effort actif32.

En conclusion, ces deux auteurs ont accordé une importance considérable à la réalité musicale apportant par là même une grande richesse dans leurs réflexions. Ces deux avis, malgré leurs éducations, leurs professions et univers musicaux différents, constituent une façon de permettre ou non, une transmission de la pensée musicale. Ces points de vue sont là pour indiquer qu'il faut surtout retourner à la musique. Or, un discours reste quand même nécessaire pour nous éclairer. C'est à la fois un engagement éthique et pratique. Un homme s'affirme et affirme ses idées; des valeurs et des sentiments à travers un discours et permet par là de faire partager, progresser les individus 
auxquels il s'adresse. Mais, l'apparence du discours est différente de son efficacité, de ce qu'il dit vraiment, de ce qu'il est capable de dévoiler, de sa véritable valeur et de sa crédibilité. Parler sur la musique ou de la musique est un autre moyen, en dehors de la musique elle-même, de rassembler, d'apporter un message et parfois même une vérité. Mais, le discours aura beau faire, il ne rejoindra jamais la chose elle-même. Le discours s'épuise en s'exprimant. II ne débouche pas sur une aporie mais laisse encore une place à l'interprétation, à la création. On comprend mieux que le discours de Vladimir Jankélévitch ne vise pas à imposer un savoir qui réduirait toute liberté de créativité, mais soit une façon d'admettre qu'il reste "de la place». Sans doute est-ce pour cela qu'il a créé le «je-ne-saisquoi» à la fois évident, fuyant, avec sa perfection et sa profondeur, compréhensible pour tous, point commun de notre expérience mais insaisissable... permettant à son discours de déboucher sur le mystère des données non révélées. Et, Igor Stravinsky ressent et exprime à peu près la même chose sur le mystère de la musique quand il écrit que la musique n'est pas autre chose que ce qu'elle est33. Finalement, dans une partition est contenu le style du compositeur qui est, selon Igor Stravinsky : «la façon particulière dont un auteur ordonne ses concepts et parle la langue de son métier. La langue est l'élément commun aux compositeurs d'une école ou d'une époque déterminée..." et qui s'ancre dans la tradition ${ }^{34}$. Mais, ce qui fait l'originalité d'une interprétation, c'est d'informer avec les éléments du passé, de façon vivante, le présent. L'interprète détient les clés d'une nouvelle redistribution du message. II est un relais, s'abstenant de corrections, de changements, d'ajouts, accédant au contenu symbolique d'une œuvre et apportant la plus grande lisibilité du sens. L'interprète prend une importance considérable et répond à la nature même de la musique supposant un créateur, un compositeur, le silence du néant et un musicien ${ }^{35}$. La bonne compréhension de la partition par un interprète est un certain équilibre entre une approche rationnelle du texte et la sensibilité, l'intuition et le talent de celui qui la porte à l'audition, au concert ou au récital. C'est vraisemblablement la dimension humaine qui est oubliée par Igor Stravinsky. Cette dimension humaine, sensible, émotive est pourtant indissociable de la dimension intellectuelle, ingrédient de toutes bonnes interprétations. Sans le formuler aussi explicitement, Vladimir Jankélévitch est conscient qu'un texte musical est une source de questionnement renvoyant ainsi l'homme à la question du sens, et justifiant le fait 
qu'une interprétation est toujours à remettre en question, à discuter et est sans fin. En conséquence, il n'existe pas de vraies ou de fausses interprétations, mais des interprétations fortes ou faibles. II serait intéressant alors de se pencher sur la vie même de l'interprétation se rapprochant de la vie, elle-même interprétative. II serait bon également de se pencher sur la méthode utilisée par l'interprète, son questionnement constant avec le texte, sa recherche de la source de l'interrogation elle-même où viennent se mêler son talent et l'idée de beauté, ses pulsions, ses désirs, ses volontés... et surtout son vécu intimement lié au contexte social, idéologique et historique dans lequel il évolue. L'essence d'une interprétation forte ne touche-t-elle pas au plus profond l'existence de tout être humain et ne crée-t-elle pas par là même une cohésion sociale?

\section{Emmanuelle Corbel Université de la Culture Permanente France}

1. Vladimir Jankélévitch : philosophe français né à Bourges en 1903 et décédé à Paris en 1985.

2. Igor Stravinsky : compositeur russe, naturalisé français puis américain, né à Oraniembaum près de Saint-Pétersbourg en 1882 et décédé à New York en 1971.

3. Igor Stravinsky, Chroniques de ma vie, Paris, Denoël, 1962 : ouvrage mêlant à la fois le genre biographique et tentant de rectifier, selon lui, le message qu'il tenait à faire passer dans ses œuvres. Igor Stravinsky, Poétique musicale, Paris, La Flûte de Pan coll. «Études musicales", Paris, 1945 : est un ouvrage qui rassemble les cours que le compositeur a donnés à P'Université de Harvard en 1939, année où après avoir vécu en France, il s'installa aux États-unis en répondant à l'invitation de cette prestigieuse université. Nous nous référons également à l'ouvrage des entretiens accordés à Robert Craft traduit en français dont les références sont les suivantes : Igor Stravinsky et Robert Craft, Souvenirs et commentaires, Paris, Gallimard, 1963.

4. Valérie Dufour, "La Poétique musicale de Stravinsky : un manuscrit inédit de Souvtchinsky" in Revue de Musicologie, tome 89, $n: 2,2003$, p. 373-392.

5. Igor Stravinsky et Robert Craft, op.cit., p. 88-89. Igor Stravinsky reconnaît l'aide précieuse de Paul Valéry dans la correction et l'élaboration de ses conférences pour l'Université de Harvard et pour "La Poétique de la Musique". L'article de Valérie Dufour mentionne également l'aide pour ses écrits de deux collaborateurs : Pierre Souvtchinsky (1892-1985) russe émigré de la révolution bolchevique, auteur de nombreux écrits sur les musiciens et Roland-Manuel (Roland Alexis Manuel Lévy dit) musicologue français (1891-1966), tous deux amis de Stravinsky. 


\section{Emmanuelle Corbel}

6. Il est à noter concernant l'originalité de ce penseur et l'intemporalité de ses idées et réflexions, qu'il n'a jamais succombé à la facilité d'écouter des enregistrements et n'a jamais cité des noms d'interprètes en référence ou pour illustrer sa pensée. Là est peut-être aussi l'une de ses positions radicales, mais néanmoins aussi inattendue, originale, qu'intéressante, séduisante.

7. Cette révélation comprend à la fois la technique musicale (mélodies, harmonies, phrasés...), le langage musical et la temporalité musicale, mais elle se situe aussi bien au delà.

8. Dans l'ensemble de son œuvre, on trouve un grand nombre de compositeurs français, de compositeurs russes et de compositeurs espagnols... soit un total de plus de cent vingt compositeurs.

9. Emmanuelle Corbel, Vladimir Jankélévitch et le mystère de l'interprétation dans la musique française de la fin $d u X I X^{\ominus}$ et du début du XX列 siècle, Nancy, Université de Nancy 2, 2004, où dans la première partie sont étudiés et distingués ces différents termes faisant partie de la terminologie propre à Vladimir Jankélévitch.

10. Ibid., j'y analyse le mystère dans le langage verbal et musical, dans la création, la composition musicale et enfin dans l'exécution musicale, différents moments où l'interprétation peut se situer, peut entrer en jeu, intervenir.

11. Olivier Hyafil, «À l'écoute de Vladimir Jankélévitch» in Diapason n² 243 , Octobre 1979.

12. Vladimir Jankélévitch, La Musique et l'ineffable, Paris, Le Seuil, 1983, p. 92.

13. Ibid., 78-83. La musique est selon lui, «un espressivo inexpressif»; elle suggère après coup, exprime l'inexprimable à l'infini.

14. Vladimir Jankélévitch , L'Irréversible et la nostalgie, Paris, Flammarion, 1974 et Vladimir Jankélévitch et Béatrice Berlowitz, Quelque part dans l'inachevé, Paris, Gallimard, 1978.

15. Igor Stravinsky, Chroniques de ma vie, p. 69-70 où il expose clairement son avis sur la question : "Car je considère la musique par son essence, impuissante à exprimer quoi que ce soit : un sentiment, une attitude, un état psychologique, un phénomène de la nature, etc. L'expression n'a jamais été la propriété immanente de la musique. La raison d'être de celle-ci n'est d'aucune façon inconditionnée par celle-là. Si, comme c'est presque toujours le cas, la musique paraît exprimer quelque chose, ce n'est qu'une illusion et non une réalité", ainsi que p. 211-214. II est également souhaitable de consulter Igor Stravinsky, Poétique musicale, p. 71 où il s'interroge sur la recherche du sens ou de la vérité de la musique semblant être, pour lui, un long parcours, peut être impossible à résoudre.

16. Igor Stravinsky, Chroniques de ma vie, p. 125 : Grâce aux enregistrements effectués par la maison Pleyel, ses intentions et ses directives pour les interprètes sont conservées. Le but des ces enregistrements est de fixer ses intentions : les rapports des mouvements (tempi), les nuances à établir... Cela évite de s'égarer dans une interprétation arbitraire de son texte musical.

17. Igor Stravinsky, Poétique musicale, p. 139-141 où il insiste sur la nature de la musique supposant le créateur, le silence du néant et l'exécutant.

18. Igor Stravinsky, Chroniques de ma vie, p. 94-96 : pages où il expose ses réflexions sur l'interprète et l'interprétation, puis sur la musique contemporaine. II veut que l'interprète transmette sa pensée musicale au public sans la trahir par une interprétation personnelle et arbitraire. Selon lui, la musique doit être transmise et non pas interprétée. L'interprétation révèle plutôt la personnalité de l'interprète que 
celle de l'auteur. L'exécutant doit refléter sans l'altérer l'image du créateur. II se mesure à la faculté de voir ce qui, en fait, se trouve dans la partition et non pas, certes, à son obstination d'y chercher ce qu'il voudrait qu'il fût. On peut mettre en parallèle ces réflexions avec celles que l'on trouve dans l'ouvrage déjà cité de Igor Stravinsky et Robert Craft, p. 139-140, où il donne sa définition des vrais et des faux virtuoses venant en écho ici, et prouvant combien ce problème lui tenait à cœur.

19. Igor Stravinsky, Poétique musicale, p. 141. En ce sens, s'éclairent les durs propos émis sur l'interprétation dans Igor Stravinsky et Robert Craft, p. 142-143 où selon lui, "le mot n'est qu'un mythe». On comprend avec le recul nécessaire que cette phrase n'est en fait qu'une façon de réaffirmer son autorité en tant que compositeur afin de faire respecter le texte.

20. Ibid., p. 143.

21. Ibid., p. 149-150.

22. Ibid., p. 145.

23. Ibid., p. 146, 147.

24. Ibid., p. 151, 157-158.

25. Ibid., p. 150-151 et p.167, mais aussi dans Igor Stravinsky, Chroniques de ma vie, p. 89-92 : où pour la constitution d'un théâtre ambulant, la composition de L'Histoire du soldat, il tient compte de la part active de l'œil dans un concert.

26. Vladimir Jankélévitch a t-il écrit un traité ou un ouvrage sur l'interprétation? Non. Pourtant, au fur et à mesure que l'on entre dans cette pensée, qu'on l'approfondit, il se dégage des termes essentiels, des concepts au sein de sa pensée qui rejoignent I'interprétation. Vladimir Jankélévitch a-t-il omis ou oublié cet important concept? II semble que oui, mais seulement au premier abord car très vite cet incroyable déchiffreur et analyste de partitions dégage une véritable pensée à laquelle la musicologie a attaché jusqu'à présent trop peu d'importance.

27. Igor Stravinsky, Chroniques de ma vie, p. 47-48.

28. Cela n'est pas traité par Igor Stravinsky mais par Vladimir Jankélévitch qui est conscient qu'une relecture constante est nécessaire.

29. Igor Stravinsky, Chroniques de ma vie, p. 125 : le but de ces enregistrements est la fixation de ses intentions : les rapports des mouvements (tempi), les nuances à établir... et dans Igor Stravinsky, Poétique musicale, où dans la deuxième partie intitulée «Du phénomène musical», p. 59, Igor Stravinsky expose sa méthode pour composer. Ces pages sont à consulter car il est impossible d'aborder ici les différents éléments qui structurent le langage musical mais qui néanmoins contribuent à la perception et à l'interprétation d'une œuvre.

30. Pierre-André Taguieff, "Vladimir Jankélévitch : les apories de l'éthique et la musique de la métaphysique" in Cahiers Bernard Lazare, no 113, OctobreDécembre 1985, p. 88

31. Cf. Vladimir Jankélévitch, La Musique et l'ineffable, p. 106.

32. Cf. Igor Stravinsky, Chroniques de ma vie, p. 182-187 où déjà, à cette époque, Igor Stravinsky met en garde ses lecteurs sur le vice caché des enregistrements rendant le mélomane passif alors que la musique demande un effort actif, effort qu'effectue l'interprète. II faut aussi se référer à lgor Stravinsky, Poétique musicale, p. 71 où il explique l'attitude du mélomane en attente de tout comprendre de la musique, aussi bien lorsqu'elle lui procure de l'émotion que lorsqu'elle lui déplaît alors que la musique reste, par essence, ineffable. 